\title{
Network Traffic Flow Evolution Model Based on Disequilibrium Theory
}

\author{
Zhongxiang Huang $\mathbb{D}^{1},{ }^{1}$ Jianhui $W u\left(\mathbb{D},{ }^{1,2}\right.$ Ruqing Huang $\left(\mathbb{D},{ }^{3}\right.$ and Yan $X u(\mathbb{D})^{2}$ \\ ${ }^{1}$ School of Traffic and Transportation Engineering, Changsha University of Science and Technology, Changsha 410114, China \\ ${ }^{2}$ School of Information Science and Technology, Hunan Institute of Science and Technology, Yueyang 414006, China \\ ${ }^{3}$ School of Traffic and Transportation Engineering, Central South University, Changsha 410075, China
}

Correspondence should be addressed to Jianhui Wu; wjh_hnist@163.com

Received 25 March 2018; Accepted 19 July 2018; Published 9 August 2018

Academic Editor: Dong Ngoduy

Copyright ( 2018 Zhongxiang Huang et al. This is an open access article distributed under the Creative Commons Attribution License, which permits unrestricted use, distribution, and reproduction in any medium, provided the original work is properly cited.

\begin{abstract}
The disequilibrium theory in economics is used to depict the network traffic flow evolution process from disequilibrium to equilibrium. Three path choice behavior criteria are proposed, and the equilibrium traffic flow patterns formed by these three criteria are defined as price regulation user equilibrium, quantity regulation user equilibrium, and price-quantity regulation user equilibrium, respectively. Based on the principle of price-quantity regulation user equilibrium, the method of network tatonnement process is used to establish a network traffic flow evolution model. The unique solution of the evolution model is proved by using Picard's existence and uniqueness theorem, and the stability condition of the unique solution is derived based on stability theorem of nonlinear system. Through numerical experiments, the evolution processes of network traffic flow under different regulation modes are analyzed. The results show that all the single price regulation, single quantity regulation, and price-quantity regulation can simulate the evolution process of network traffic flow. Price-quantity regulation is the combination of price regulation user equilibrium and quantity regulation user equilibrium, which thus can simulate the evolution process of network traffic flow with multiple user class.
\end{abstract}

\section{Introduction}

Network traffic flow evolution model is mainly used to study the network traffic flow evolution process and traffic fluctuations, which is an important part of traffic network design and traffic management strategy optimization. In order to give a more accurate and meticulous depiction of the formation and evolution of network traffic flow, it is necessary to start with the traveler's travel choice behavior. There are many factors affecting a traveler's travel choice behavior and the decision-making process is complex. Since the establishment of two important principles of user equilibrium and system optimization put forward by Wardrop [1], scholars have done a lot of research on this basis. Smith [2] supposed that travelers would consider changing their routes in order to obtain lower travel time and proposed a continuous dayto-day evolution model. Friesz et al. [3] studied day-today adjustment process of network traffic flow and analyzed the impact of information integrity for network traffic flow. Zhang and Nagurney [4] proposed a deterministic dayto-day evolution model by using the method of projected dynamical system. Wei et al. [5] presented a day-to-day route choice model by using reinforcement learning and multiagent simulation.

At present, the theories used to study the evolution model of network traffic flow mainly include random utility theory (e.g., Wen et al. [6] and Chen and Pan [7]), expected utility theory (e.g., Savage [8] and Blavatskyy [9]), prospect theory (e.g., Li et al. [10], Liu [11], and Wang et al. [12]), regret theory (e.g., Chorus [13] and Ramos et al. [14]), and disequilibrium theory (e.g., Zhang and Monden [15] and Huang et al. [16]). Random utility theory and expected utility theory are based on the assumption of traveler's complete rationality, and they are widely applied in the analysis of travel choice behavior and the description of traffic flow evolution law. In the face of many uncertain factors, people's judgment and 
decision-making behavior are not completely rational, but greatly influenced by personal habit preference, risk attitude, and so on, so it is bounded rationality. Both prospect theory and regret theory are based on the hypothesis of bounded rationality and can better describe the evolution process of network traffic flow in uncertain scenarios. Avineri [17] has applied the prospect theory to stochastic user equilibrium and studied the influence of reference point on stochastic user equilibrium. Based on regret theory, scholars like Chorus [18] proposed a stochastic regret minimum model based on the assumption that the traveler's choice of one path depends only on the regret value obtained by comparing the path with the best path in other alternative paths. As a matter of fact, travel choice is usually repeated daily decision, while prospect theory and regret theory mainly involve one decision and do not take the travelers' learning and behavior adjustment of decision results into consideration.

The disequilibrium theory regards road network system as an economic market, the potential traveler of road network as the travel demand-side, and the road network itself as the travel supply-side. The decision behaviors of travelers are explained from two factors, price, and quantity, so as to describe the dynamic evolution of traffic flow. Huang et al. [19] proposed an idea of disequilibrium transportation planning based on disequilibrium theory and believed that travelers will not only consider the travel time (price) of the current route, but also the traffic flow (quantity) obtained from traffic information and historical experience. In the actual traffic management, congestion charges (e.g., Wu et al. [20], Chiou and Fu [21], and Jia et al. [22]) and parking charges (e.g., Washbrook et al. [23] and Simićević et al. [24]) are to change the travel decisions of some travelers through typically price regulation. Ramp control (e.g., Hasan et al. [25] and Frejo and Camacho [26]) and public transport departure frequency optimization (e.g., Berrebi et al. [27] and Wang et al. [28]) are to change the travel decision of some travelers through typically quantity regulation, so travelers are subject to the double constraints of price and quantity. Based on this, this paper assumes that some travelers choose the lowest time path, and some travelers choose the most comfortable path. A concept of path-residual capacity is proposed to describe the driving comfort. Based on disequilibrium theory, pathtravel time is regarded as price, path-residual capacity is regarded as quantity, and the influence of price-quantity on the distribution of traffic flow on the road network is also analyzed.

The remainder of this paper is organized as follows. In the next section, we propose three path choice behavior criteria based on disequilibrium theory. Section 3 establishes a network traffic flow evolution model. Section 4 proves the existence, uniqueness, and stability of solution to the evolution model. Section 5 verifies the evolution process and stable state of network traffic flow under different regulation modes. Section 6 is the conclusions of this research.

\section{Path Choice Behaviors}

The different criterion of path choice naturally leads to the different distribution of network traffic flow. This paper proposes three types of path choice behavior criterion based on disequilibrium theory and defines the stable state of network traffic flow formed by these three criteria as price regulation user equilibrium, quantity regulation user equilibrium, and price-quantity regulation user equilibrium, respectively.

2.1. Price Regulation User Equilibrium. Suppose that travelers have complete travel time (price) information and choose travel path according to the minimum travel time. We call this kind of travelers' path choice behavior to follow price regulation completely.

The relationship between travel time $c_{p}$ on path $p$ and travel time $c_{a}$ on link $a$ can be written as

$$
c_{p}(h(t))=\sum_{a \in A} \delta_{a p} c_{a}(f(t))
$$

where $A$ represents the set of links, $f_{a}(t)$ is the traffic flow on link $a$ at time $t, h_{p}(t)$ is the traffic flow on path $p$ at time $t$, and $\delta_{a p}$ is a link-path incidence relationship, specifically $\delta_{a p}=1$ if $a \in p$ and $\delta_{a p}=0$ otherwise.

We define price regulation user equilibrium as the travel time on all used paths on OD pair is equal and is less than or equal to the travel time on any unused paths. It can be expressed as

$$
c_{p}(h(t)) \begin{cases}=\mu_{i j}(t), & \text { if } h_{p}(t)>0 \\ \geq \mu_{i j}(t), & \text { if } h_{p}(t)=0\end{cases}
$$

Subject to

$$
\begin{array}{r}
\sum_{p \in P_{i j}} h_{p}(t)=T_{i j}(\mu(t)), \quad \forall i \in N_{O}, \quad j \in N_{D} \\
h_{p}(t) \geq 0, \quad \forall p \in P_{i j}, \quad i \in N_{O}, \quad j \in N_{D}
\end{array}
$$

where $N_{O}$ represents the set of nodes which are trip origins, $N_{D}$ is the set of nodes which are trip destinations, $P_{i j}$ is the set of paths on OD pair $(i, j), P=\left(P_{i j}: i \in N_{O}, j \in N_{D}\right)$, $\mu_{i j}(t)$ is the minimum travel time on OD pair $(i, j)$ at time $t$, and travel demand $T_{i j}(\mu(t))$ is a function of $\mu_{i j}(t)$.

Evidently, the price regulation user equilibrium is Wardrop's user equilibrium.

2.2. Quantity Regulation User Equilibrium. In the process of travel decision-making, travelers may not always choose travel path according to the minimum travel time even they have got complete traffic information. For example, from the northeast corner of North 3rd Ring Road in Beijing to the Beijing Capital International Airport, there are two alternative routes: Airport Expressway and Jingcheng Expressway. Because Jingcheng Expressway has smaller vehicle flow, better driving comfort, and higher travel time reliability, some travelers would like to choose Jingcheng Expressway rather than Airport Expressway with high vehicle flow and short travel time.

Suppose that travelers cannot get travel time information or do not choose travel path according to the travel time, but 
choose travel path according to the vehicle flow (quantity) on the optional paths. We call this kind of travelers' path choice behavior to follow quantity regulation completely.

This paper uses the method of residual capacity to define the quantity regulation variable of vehicle flow on the link. The more the residual capacity, the better the drive comfort, and the higher the travel time reliability. We express $K_{a}$ as the capacity on link $a, R E V_{a}$ as the residual capacity on link a, $R E V_{a}(f(t))=K_{a}-f_{a}(t)$; hence the residual capacity $R E V_{p}$ on path $p$ can be written as

$$
R E V_{p}(h(t))=\min _{a \in p}\left\{R E V_{a}(f(t))\right\}
$$

We define quantity regulation user equilibrium as the residual capacity on all used paths on OD pair is equal and is greater than or equal to the residual capacity on any unused paths. It can be expressed as

$$
R E V_{p}(h(t)) \begin{cases}=v_{i j}(t), & \text { if } h_{p}(t)>0 \\ \leq v_{i j}(t), & \text { if } h_{p}(t)=0\end{cases}
$$

subject to

$$
\begin{array}{r}
\sum_{p \in P_{i j}} h_{p}(t)=T_{i j}(v(t)), \quad \forall i \in N_{O}, j \in N_{D} \\
h_{p}(t) \geq 0, \quad \forall p \in P_{i j}, \quad i \in N_{O}, \quad j \in N_{D}
\end{array}
$$

where $v_{i j}(t)$ is the maximum residual capacity on OD pair $(i, j)$ at time $t$ and travel demand $T_{i j}(v(t))$ is a function of $v_{i j}(t)$.

2.3. Price-Quantity Regulation User Equilibrium. Based on the price-quantity regulation principle of disequilibrium theory in economics, it is assumed that travelers choose travel path according to the comprehensive travel cost on the optional paths. We define the comprehensive travel cost $\gamma_{p}$ on path $p$ as

$$
\gamma_{p}(h(t))=\lambda c_{p}(h(t))-(1-\lambda) R E V_{p}(h(t))
$$

where $\lambda \in[0,1]$ is weight factor, reflecting the sensitivity of travelers to travel time $c_{p}$ and residual capacity $R E V_{p}$.

We define the minimum comprehensive travel cost $\pi_{i j}$ on OD pair $(i, j)$ as

$$
\pi_{i j}(t)=\lambda \mu_{i j}(t)-(1-\lambda) v_{i j}(t)
$$

We define price-quantity regulation user equilibrium as the comprehensive travel cost on all used paths on OD pair is equal and is less than or equal to the comprehensive travel cost on any unused paths. It can be expressed as

$$
\begin{aligned}
& \lambda c_{p}(h(t))-(1-\lambda) \\
& \cdot R E V_{p}(h(t)) \begin{cases}=\lambda \mu_{i j}(t)-(1-\lambda) v_{i j}(t), & \text { if } h_{p}(t)>0 \\
\geq \lambda \mu_{i j}(t)-(1-\lambda) v_{i j}(t), & \text { if } h_{p}(t)=0\end{cases}
\end{aligned}
$$

subject to

$$
\begin{gathered}
\sum_{p \in P_{i j}} h_{p}(t)=T_{i j}(\mu(t), v(t)), \quad \forall i \in N_{O}, j \in N_{D} \\
h_{p}(t) \geq 0, \quad \forall p \in P_{i j}, \quad i \in N_{O}, \quad j \in N_{D}
\end{gathered}
$$

where travel demand $T_{i j}(\mu(t), v(t))$ is a function of $\mu_{i j}(t)$ and $v_{i j}(t)$.

For the above user equilibrium, if $\lambda=1$, travelers choose travel path only according to travel time, and price-quantity regulation user equilibrium becomes single price regulation user equilibrium; if $\lambda=0$, travelers choose travel path only according to residual capacity, and price-quantity regulation user equilibrium becomes single quantity regulation user equilibrium.

\section{Network Traffic Flow Evolution Model}

According to price-quantity regulation user equilibrium, this paper uses the method of network tatonnement process to simulate travelers' path choice behavior and constructs a network traffic flow evolution model.

To give a mathematical state of this model we express the excess travel time $E T C_{p}$ on path $p$ as

$$
\operatorname{ETC}_{p}\left(\mu_{i j}(t), h(t)\right)=c_{p}(h(t))-\mu_{i j}(t)
$$

The excess residual capacity $E T V_{p}$ on path $p$ is expressed as

$$
\operatorname{ETV}_{p}\left(v_{i j}(t), h(t)\right)=R E V_{p}(h(t))-v_{i j}(t)
$$

We define excess comprehensive travel cost $E C C_{p}$ on path $p$ as comprehensive travel cost $\gamma_{p}$ minus minimum comprehensive travel cost $\pi_{i j}$, that is, formula (7) minus (8). It can be expressed as

$$
\begin{aligned}
E \operatorname{EC}_{p} & \left(\mu_{i j}(t), v_{i j}(t), h(t)\right) \\
= & \lambda\left[c_{p}(h(t))-\mu_{i j}(t)\right] \\
& -(1-\lambda)\left[R E V_{p}(h(t))-v_{i j}(t)\right]
\end{aligned}
$$

Evidently

$$
\begin{aligned}
\operatorname{ECC}_{p}\left(\mu_{i j}(t), v_{i j}(t), h(t)\right) \\
=\lambda \operatorname{ETC}_{p}\left(\mu_{i j}(t), h(t)\right) \\
\quad-(1-\lambda) \operatorname{ETV}_{p}\left(v_{i j}(t), h(t)\right)
\end{aligned}
$$

When excess comprehensive travel cost $E C C_{p}$ at time $t$ is positive (comprehensive travel cost more than minimum comprehensive travel cost), path flow $h_{p}$ will decrease because some travelers will automatically move to the lower comprehensive travel cost path; otherwise path flow $h_{p}$ will 
increase. To this end, the adjustment principle of $h_{p}$ and $E C C_{p}$ can be expressed as

$$
\begin{aligned}
& h_{p}(t+\Delta t)=h_{p}(t)-\beta E C C_{p}\left(\mu_{i j}(t), v_{i j}(t), h(t)\right) \\
& =h_{p}(t)-\beta\left[\lambda \operatorname{ETC}_{p}\left(\mu_{i j}(t), h(t)\right)\right. \\
& \left.-(1-\lambda) \operatorname{ETV}_{p}\left(v_{i j}(t), h(t)\right)\right], \quad \forall \beta>0
\end{aligned}
$$

Considering $h_{p} \geq 0$, formula (15) can be rewritten as

$$
\begin{aligned}
& h_{p}(t+\Delta t)=\left\{h_{p}(t)-\beta\left[\lambda E T C_{p}\left(\mu_{i j}(t), h(t)\right)\right.\right. \\
& \left.\left.-(1-\lambda) \operatorname{ETV}_{p}\left(v_{i j}(t), h(t)\right)\right]\right\}_{+}
\end{aligned}
$$

where $\{z\}_{+}=\max (0, z)$. Supposing that $h_{p}$ is a continuous differentiability function of $t$, we have

$$
\begin{aligned}
\frac{d h_{p}(t)}{d t} & =\lim _{\Delta t \rightarrow 0} \frac{h_{p}(t+\Delta t)-h_{p}(t)}{\Delta t} \\
& \approx \eta_{p}\left[h_{p}(t+\Delta t)-h_{p}(t)\right], \quad \forall \eta_{p}>0
\end{aligned}
$$

Formula (16) into (17), we also have

$$
\begin{gathered}
\frac{d h_{p}(t)}{d t}=\eta_{p}\left[\left\{h_{p}(t)-\beta\left[\lambda E T C_{p}\left(\mu_{i j}(t), h(t)\right)\right.\right.\right. \\
\left.\left.\left.-(1-\lambda) \operatorname{ETV}_{p}\left(v_{i j}(t), h(t)\right)\right]\right\}_{+}-h_{p}(t)\right]
\end{gathered}
$$

The excess travel demand $E T D_{i j}$ on OD pair $(i, j)$ is expressed as

$$
\begin{aligned}
& \operatorname{ETD}_{i j}\left(\mu(t), v(t), h_{p}(t)\right) \\
& \quad=T_{i j}(\mu(t), v(t))-\sum_{p \in P_{i j}} h_{p}(t)
\end{aligned}
$$

When excess travel demand $E T D_{i j}$ at time $t$ is positive, the minimum travel time will increase and the maximum residual capacity will decrease to reduce the potential travel demand; otherwise the minimum travel time will decrease and the maximum residual capacity will increase. To this end, the adjustment principle of $E T D_{i j}, \mu_{i j}$, and $v_{i j}$ can be expressed as

$$
\begin{array}{r}
\mu_{i j}(t+\Delta t) \\
=\left\{\mu_{i j}(t)+\alpha E T D_{i j}\left(\mu(t), v(t), h_{p}(t)\right)\right\}_{+}, \\
\forall \alpha>0 \\
v_{i j}(t+\Delta t)=\left\{v_{i j}(t)-\vartheta \operatorname{ETD}_{i j}\left(\mu(t), v(t), h_{p}(t)\right)\right\}_{+}, \\
\forall \vartheta>0
\end{array}
$$

Supposing that $\mu_{i j}$ is a continuous differentiability function of $t$, we know

$$
\frac{d \mu_{i j}(t)}{d t} \approx \kappa_{i j}\left[\mu_{i j}(t+\Delta t)-\mu_{i j}(t)\right], \quad \forall \kappa_{i j}>0
$$

Formula (20) into (21), we have

$$
\begin{aligned}
& \frac{d \mu_{i j}(t)}{d t} \\
& \quad=\kappa_{i j}\left[\left\{\mu_{i j}(t)+\alpha E T D_{i j}\left(\mu(t), v(t), h_{p}(t)\right)\right\}_{+}\right. \\
& \left.-\mu_{i j}(t)\right]
\end{aligned}
$$

Supposing that $v_{i j}$ is a continuous differentiability function of $t$, we also have

$$
\begin{aligned}
& \frac{d v_{i j}(t)}{d t} \\
& \quad=\omega_{i j}\left[\left\{v_{i j}(t)-\vartheta E T D_{i j}\left(\mu(t), v(t), h_{p}(t)\right)\right\}_{+}\right. \\
& \left.-v_{i j}(t)\right], \quad \forall \omega_{i j}>0
\end{aligned}
$$

Furthermore, we impose the initial conditions $\mu(t=0)=$ $\mu^{0}, v(t=0)=v^{0}$ and $h(t=0)=h^{0}$. Using formulas (18) (23) (24), network traffic flow evolution model can be expressed as

$$
\begin{aligned}
& \frac{d \mu(t)}{d t}=\kappa\left[\{\mu(t)+\alpha \operatorname{ETD}(\mu(t), v(t), h(t))\}_{+}\right. \\
& -\mu(t)] \\
& \frac{d v(t)}{d t}=\omega\left[\{v(t)-\vartheta \operatorname{ETD}(\mu(t), v(t), h(t))\}_{+}\right. \\
& \quad-v(t)] \\
& \frac{d h(t)}{d t}=\eta[\{h(t)-\beta[\lambda \operatorname{ETC}(\mu(t), h(t)) \\
& \left.-(1-\lambda) \operatorname{ETV}(v(t), h(t))]\}_{+}-h(t)\right]
\end{aligned}
$$

where $\mu(t)=\left(\mu_{i j}(t): i \in N_{O}, j \in N_{D}\right)^{\prime}, v(t)=\left(v_{i j}(t): i \epsilon\right.$ $\left.N_{O}, j \in N_{D}\right)^{\prime}, h(t)=\left(h_{p}(t): p \in P\right)^{\prime}, E T D \equiv\left(E T D_{i j}: i \epsilon\right.$ $\left.N_{O}, j \in N_{D}\right)^{\prime}, E T C \equiv\left(E T C_{p}: p \in P\right)^{\prime}, E T V \equiv\left(E T V_{p}: p \epsilon\right.$ $P)^{\prime}, \lambda \in[0,1], \kappa=\operatorname{diag}\left(\kappa_{i j}: i \in N_{O}, j \in N_{D}\right), \omega=\operatorname{diag}\left(\omega_{i j}:\right.$ $\left.i \in N_{O}, j \in N_{D}\right), \eta=\operatorname{diag}\left(\eta_{p}: p \in P\right)$.

\section{Existence, Uniqueness, and Stability}

4.1. Existence and Uniqueness. For the convenience of analysis, we make the following definitions:

$$
x \equiv\left(\begin{array}{c}
\mu \\
v \\
h
\end{array}\right),
$$

$$
E(x) \equiv\left(\begin{array}{c}
-T(\mu, v) \\
T(\mu, v) \\
\lambda_{c}(h)-(1-\lambda) R E V(h)
\end{array}\right)
$$




$$
\begin{aligned}
F(x) & \equiv\left(\begin{array}{cc}
-E T D \\
E T D \\
\lambda E T C-(1-\lambda) E T V
\end{array}\right), \\
B & \equiv\left(\begin{array}{ccc}
0 & 0 & R^{\prime} \\
0 & 0 & -R^{\prime} \\
-\lambda R & (1-\lambda) R & 0
\end{array}\right)
\end{aligned}
$$

Network traffic flow evolution model (25) can be reexpressed as

$$
\frac{d x}{d t}=a\left[\{x-b F(x)\}_{+}-x\right]
$$

where $a=\operatorname{diag}(\kappa, \omega, \eta), b=\operatorname{diag}(\alpha, \vartheta, \beta), F(x)=E(x)+$ $B x, R=\left(r_{p}^{i j}: p \in P, i \in N_{O}, j \in N_{D}\right)$ is the path-OD pair incidence matrix, specifically $r_{p}^{i j}=1$ if path $p$ connects $\mathrm{OD}$ pair $(i, j)$ and zero otherwise.

Hypothesis 1. $c_{a}(f)$ is a positive, continuous, and strictly monotonically increasing function of $f_{a}, R E V(h)$ is a positive, continuous, and strictly monotonically decreasing function of $h$, and $T(\mu, v)$ is a continuous, bounded, and strictly monotonic function of $\mu$ and $v$.

Theorem 2. For any $x(0)$, there exists a unique solution $x^{*}$ to network traffic flow evolution model (25).

Proof. Let $Q(x)=a\left[\{x-b F(x)\}_{+}-x\right]$, then we introduce a Picard's existence and uniqueness theorem (Dupuis and Nagurney [29]).

Picard's Existence and Uniqueness. If $Q(x)$ is continuous in the feasible domain $K=\{|x-x(0)| \leq X\}$ (X is constant), and there exists constant $L, M$ with values to positive ensure that for any $x, y \in K$.

(1) $\|Q(x)-Q(y)\| \leq L\|x-y\|$, that is, local Lipschitz continuous.

(2) $\|Q(x)\| \leq M(1+\|x\|)$, that is, linearly bounded.

Then there exists a unique solution $x^{*}$ to formula (27).

We first prove that $Q(x)$ is continuous in the feasible domain $K$. According to the incidence relationship between path and link, $c(h)$ is a continuous function of $h$. Since $T(\mu, v)$ is continuous on $\mu$ and $v$, and $R E V(h)$ is continuous on $h$, then $F(x)$ is a continuous function of $x$. Evidently, $Q(x)$ is a continuous function of $x$; thus $Q(x)$ is continuous in the feasible domain $K$.

Second, we prove that $Q(x)$ satisfies the condition of local Lipschitz continuous. For any $x, y \in K$ one has

$$
\begin{aligned}
& \|Q(x)-Q(y)\| \\
& \quad \leq\|a\|\left(\left\|\{x-b F(x)\}_{+}-\{y-b F(y)\}_{+}\right\|+\|x-y\|\right) \\
& \quad \leq\|a\| \cdot\|b\| \cdot\|F(x)-F(y)\|+2\|a\| \cdot\|x-y\|
\end{aligned}
$$

Let $x^{\prime}=\left(\mu_{x}, v_{x}, h_{x}\right)^{\prime}, y^{\prime}=\left(\mu_{y}, v_{y}, h_{y}\right)^{\prime}, \ell^{\prime}=\left(\mu_{\ell}, v_{\ell}, h_{\ell}\right)^{\prime}$, $0 \leq \tau \leq 1, z=x-y, \ell=y+\tau z, H(\tau)=F(\ell)$; then

$$
\begin{aligned}
\|F(x)-F(y)\| & =\|H(1)-H(0)\|=\left\|\int_{0}^{1} \frac{d H(\tau)}{d \tau} d \tau\right\| \\
& =\left\|\int_{0}^{1} \frac{d F(\ell)}{d \ell} \frac{d \ell}{d \tau} d \tau\right\| \\
& =\left\|\frac{d F(\ell)}{d \ell}(x-y)\right\|
\end{aligned}
$$

Since $T(\mu, v)$ is bounded, then $h$ is a nonempty, bounded, and closed convex set. Moreover, according to the definition of travel time and residual capacity on the path, $c(h)$ and $R E V(h)$ are also a nonempty, bounded, and closed convex set; thus $F(x)$ is bounded. Let $L_{0}=\|d F(\ell) / d \ell\|$, formula (29) can be expressed as

$$
\|F(x)-F(y)\| \leq\left\|\frac{d F(\ell)}{d \ell}\right\| \cdot\|x-y\|=L_{0}\|x-y\|
$$

Formula (30) into (28), we have

$$
\begin{aligned}
\|Q(x)-Q(y)\| & \leq\left(L_{0}\|a\| \cdot\|b\|+2\|a\|\right)\|x-y\| \\
& =L\|x-y\|
\end{aligned}
$$

where $L=L_{0}\|a\| \cdot\|b\|+2\|a\|$. Hence $Q(x)$ is a local Lipschitz continuous function.

Finally, we prove that $Q(x)$ satisfies the condition of linearly bounded. Since $F(x)=E(x)+B x$, then $\|F(x)\| \leq$ $\|E(x)\|+\|B\| \cdot\|x\|$. Let $\ell_{a}=\|E(x)\|, \ell_{b}=\|B\|, L_{1}=\max \left\{\ell_{a}, \ell_{b}\right\}$; thus $\|F(x)\| \leq L_{1}(1+\|x\|)$. Moreover for any $x \in K$

$$
\begin{aligned}
\|Q(x)\| & \leq\|a\| \cdot\|b\| \cdot\|F(x)\|+2\|a\| \cdot\|x\| \\
& \leq\left(L_{1}\|a\| \cdot\|b\|+2\|a\|\right)(1+\|x\|) \\
& =M(1+\|x\|)
\end{aligned}
$$

where $M=L_{1}\|a\| \cdot\|b\|+2\|a\|$. Hence $Q(x)$ satisfies the condition of linearly bounded.

In conclusion, there exists a unique solution $x^{*}$ to formula (27); that is, $x^{*}$ is a unique solution to network traffic flow evolution model (25).

\subsection{Analysis of Steady State}

Theorem 3. If there exists a unique solution $x^{*}$ to network traffic flow evolution model (25) and $0<L_{0}\|b\|<2$, then the unique solution $x^{*}$ is asymptotically stable.

Proof. We introduce a stability theorem of nonlinear system (Cho and Hwang [30]).

Stability Theorem of Nonlinear System. If there exists a continuous differentiable function $V(x)$ for formula (27) and $V(x)$ satisfies the following conditions,

(1) $V\left(x^{*}\right)=0$, and $V(x)>0, \forall x>0, x \neq x^{*}$,

(2) $d V(x) / d t<0, \forall x>0, x \neq x^{*}$,

then the unique solution $x^{*}$ is asymptotically stable. 
Constructing a continuous differentiable function $V(x)$, it can be written as

$$
V(x)=\frac{1}{2}\left\|x-x^{*}\right\|^{2}
$$

According to formula (33), we know that $V(x)$ satisfies condition (1) in the stability theorem of nonlinear system, and then we only need to prove $d V(x) / d t<0$. We know

$$
\begin{aligned}
\frac{d V(x)}{d t} & =\left\|x-x^{*}\right\| \frac{d x}{d t} \\
& =\left\langle x-x^{*}, a\left[\{x-b F(x)\}_{+}-x\right]\right\rangle \\
\leq & \|a\| \cdot\left\|x-x^{*}\right\| \cdot\left\|\{x-b F(x)\}_{+}-x^{*}\right\|-\|a\| \\
& \cdot\left\|x-x^{*}\right\|^{2}
\end{aligned}
$$

$$
\begin{gathered}
\leq\left\|x-x^{*}\right\|^{2}+\|b\|^{2} \cdot\left\|F(x)-F\left(x^{*}\right)\right\|^{2} \\
-2\|b\|\left\langle F(x)-F\left(x^{*}\right), x-x^{*}\right\rangle
\end{gathered}
$$

Formula (30) into (35), we have

$$
\left\|\{x-b F(x)\}_{+}-x^{*}\right\| \leq\left|1-L_{0}\|b\|\right| \cdot\left\|x-x^{*}\right\|
$$

Formula (36) into (34), we know

$$
\begin{aligned}
\frac{d V(x)}{d t} & \leq\|a\|\left(\left|1-L_{0}\|b\|\right|-1\right)\left\|x-x^{*}\right\|^{2} \\
& =2\|a\|\left(\left|1-L_{0}\|b\|\right|-1\right) V(x)
\end{aligned}
$$

Let $\sigma=\|a\|\left(1-\left|1-L_{0}\|b\|\right|\right)$; formula (37) can be reexpressed as

$$
\frac{d V(x)}{d t} \leq-2 \sigma V(x)
$$

Since $0<L_{0}\|b\|<2$, then $\sigma=\|a\|\left(1-\left|1-L_{0}\|b\|\right|\right)>0$. In addition, since $V(x)>0$ for $x \neq x^{*}$, then $-2 \sigma V(x)<0$; thus $d V(x) / d t<0$. Hence, the unique solution $x^{*}$ of network traffic flow evolution model (25) is asymptotically stable.

\section{Calculations and Analysis of Numerical Example}

5.1. Test Road Network. A road network with nine nodes and twelve links illustrated as Figure 1 is used to show the numerical results of this established model. There is only one OD pair $(1,2)$ which is connected by six paths. The incidence relationship between path and link is shown in Table 1 .

In this numerical example, we will make use of traditional BPR link travel time functions of the following form:

$$
c_{a}(f)=c_{0}\left[1+0.15\left(\frac{f_{a}}{K_{a}}\right)^{4}\right]
$$

TABLE 1: Relationship between path and link.

\begin{tabular}{ccc}
\hline OD Pair & Path No. & Links in Path \\
\hline \multirow{4}{*}{$(1,2)$} & 1 & $1,2,5,10$ \\
& 2 & $1,4,7,10$ \\
& 3 & $1,4,9,12$ \\
& 4 & $3,6,7,10$ \\
& 5 & $3,6,9,12$ \\
6 & $3,8,11,12$ \\
\hline
\end{tabular}

TABLE 2: Parameters of link travel time function.

\begin{tabular}{lcccccccccccc}
\hline Link No. & 1 & 2 & 3 & 4 & 5 & 6 & 7 & 8 & 9 & 10 & 11 & 12 \\
\hline $\mathcal{c}_{0}$ & 30 & 20 & 35 & 10 & 20 & 10 & 30 & 20 & 30 & 30 & 20 & 30 \\
$K_{a}$ & 100 & 45 & 105 & 90 & 80 & 150 & 150 & 80 & 150 & 150 & 80 & 150 \\
\hline
\end{tabular}

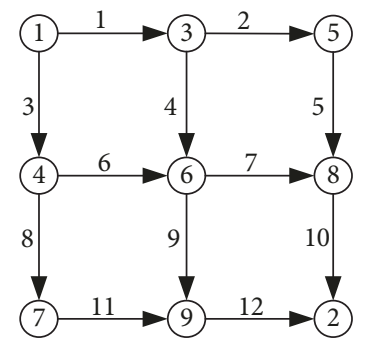

FIGURE 1: Test road network.

where $c_{0}$ is the free flow travel time on link $a$ and $K_{a}$ is the capacity on link $a$. The link travel time function parameters for this network are shown in Table 2 .

According to disequilibrium market model (Browne [31] and Huang et al. [32]), we suppose that travel demand $T_{i j}(\mu(t), v(t))$ can be expressed as

$$
T_{i j}(\mu(t), v(t))=\phi_{i j}^{\prime} X_{i j}+\varphi_{i j} \mu_{i j}(t)+\psi_{i j} v_{i j}(t)
$$

where $X_{i j}$ is the exogenous variable vector on OD pair $(i, j)$, $\phi_{i j}$ is the influence coefficient vector of $X_{i j}, \varphi_{i j}$ is the influence coefficient of $\mu_{i j}$ and $\varphi_{i j} \leq 0$, and $\psi_{i j}$ is the influence coefficient of $v_{i j}$ and $\psi_{i j} \geq 0$. In order to better compare the properties of three models, we suppose that travel demand $T_{12}(\mu(t), v(t))$ is fixed and $T_{12}(\mu(t), v(t))=80$.

5.2. Model Calculation and Evolution Process Exhibition. Travelers choose their traveling paths in a variety of manners in the real traffic process that assuming all travelers follow unified criteria to choose traveling paths is not realistic. The most important characteristic of the evolution model (25) is the ability to simulate the evolution process of network traffic flow under different circumstances. If the travelers choose their paths simply according to the travel time $(\lambda=1)$, the evolution model will become the single price regulation model and finally turns into the well-known Wardrop's user equilibrium flow pattern. If travelers choose their paths simply according to the residual capacity $(\lambda=0)$, the evolution model will be the single quantity regulation model, 


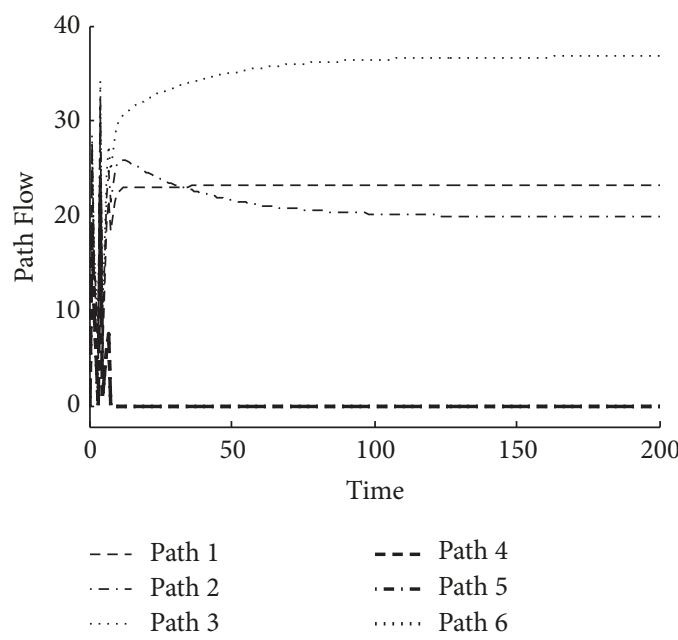

(a) Dynamic regulation process

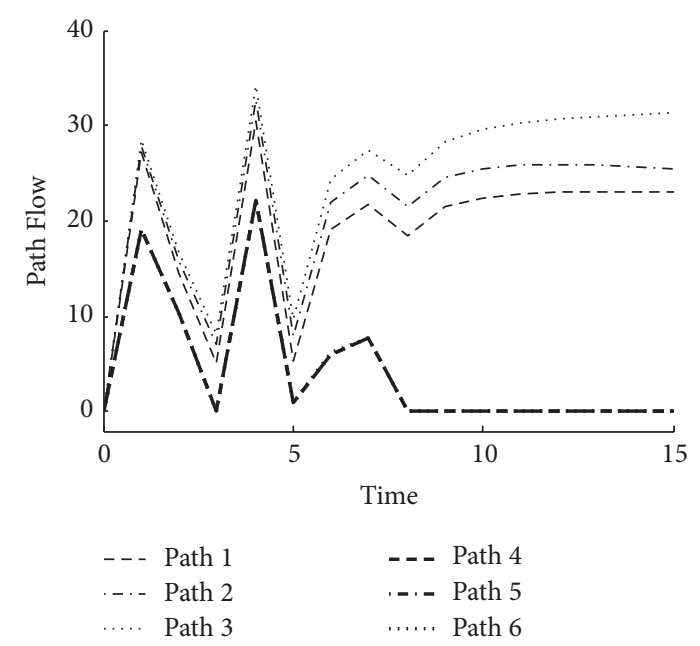

(b) Partial magnification for dynamic regulation process

FIGURE 2: Evolutionary trajectories under single price regulation.

TABLE 3: Network equilibrium results under single price regulation.

\begin{tabular}{lccc}
\hline Path No. & Path Flow & Travel Time & Residual Capacity \\
\hline 1 & 23.3085 & 102.1118 & 20 \\
2 & 19.9299 & 102.1118 & 20 \\
3 & 36.7616 & 102.1118 & 20 \\
4 & 0 & 105.0325 & 105 \\
5 & 0 & 105.0325 & 105 \\
6 & 0 & 105.0162 & 80 \\
\hline
\end{tabular}

and a new user equilibrium flow pattern can be obtained when the system is stable that the residual capacity on all used paths on OD pair are equal. If the travelers take account both travel time and residual capacity when they choose their paths $(0<\lambda<1)$, the evolution model is a price-quantity regulation model and it will become a pricequantity regulation user equilibrium flow pattern that the comprehensive travel cost on all used paths on OD pairs are equal.

Next, we will calculate and analyze these models including single price regulation model, single quantity regulation model, and price-quantity regulation model. To satisfy the stable conditions, we choose $\alpha=0.05, \vartheta=0.05, \beta=0.1$, and $\kappa=\omega=\eta=100$. Supposing that the evolution time $t \in[0,200]$, iterative step $\Delta t=0.01$, and weight factor $\lambda=0.8$ in the price-quantity model, the results of calculation are as follows.

\section{(1) Single Price Regulation. See Table 3 and Figure 2.}

(2) Single Quantity Regulation. See Table 4 and Figure 3.

\section{(3) Price-Quantity Regulation. See Table 5 and Figure 4.}

5.3. Result Analysis. It is not difficult to see from the Figures 2-4 that the evolution process of the path flow can be ideally
TABLE 4: Network equilibrium results under single quantity regulation.

\begin{tabular}{lccc}
\hline Path No. & Path Flow & Travel Time & Residual Capacity \\
\hline 1 & 0 & 100.0623 & 45 \\
2 & 16.2500 & 100.1017 & 57.5 \\
3 & 16.2500 & 100.1259 & 57.5 \\
4 & 18.5156 & 105.2514 & 57.5 \\
5 & 18.5156 & 105.2756 & 57.5 \\
6 & 10.4688 & 105.2589 & 57.5 \\
\hline
\end{tabular}

simulated by any of the three regulation models. Though each path flow will eventually reach a steady state, there are obvious differences between the evolution process and the final stable state of the three regulation models. Comparing Figures 2, 3, and 4, the fluctuation of the single price regulation model is the largest among three models and each path flow varies from disequilibrium state to equilibrium state driven by the price regulation. The fluctuation of the single quantity regulation is the smallest with the fastest convergence rate, and the price-quantity regulation model is between the other two models.

Results of the single price regulation in Table 3 show that travel time on all used paths are equal and not larger than the unused paths, which verifies that the price regulation user equilibrium is the classic Wardrop's user equilibrium. The results of the single quantity regulation in Table 4 show that the residual capacity on all used paths are not less than that of the unused paths which is to say there are more free driving opportunities for the used paths than the unused paths. The results of the price-quantity regulation in Table 5 is a combination of the price regulation user equilibrium and quantity regulation user equilibrium, where the comprehensive travel cost on all used paths are not larger than the unused paths. 
TABLE 5: Network equilibrium results under price-quantity regulation.

\begin{tabular}{lcccc}
\hline Path No. & Path Flow & Travel Time & Residual Capacity & Comprehensive Travel Cost \\
\hline 1 & 0 & 100.1611 & 45 & 71.1289 \\
2 & 23.1186 & 100.2517 & 47.7545 & 70.6505 \\
3 & 19.1269 & 100.2517 & 47.7545 & 70.6505 \\
4 & 14.4876 & 105.1245 & 67.2455 & 70.6505 \\
5 & 10.4960 & 105.1245 & 67.2455 & 70.6505 \\
6 & 12.7709 & 105.1204 & 67.2291 & 70.6505 \\
\hline
\end{tabular}

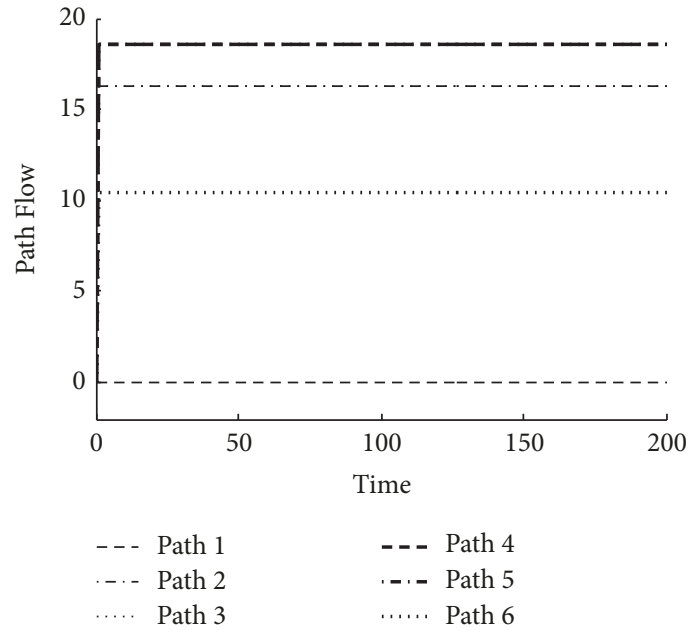

(a) Dynamic regulation process

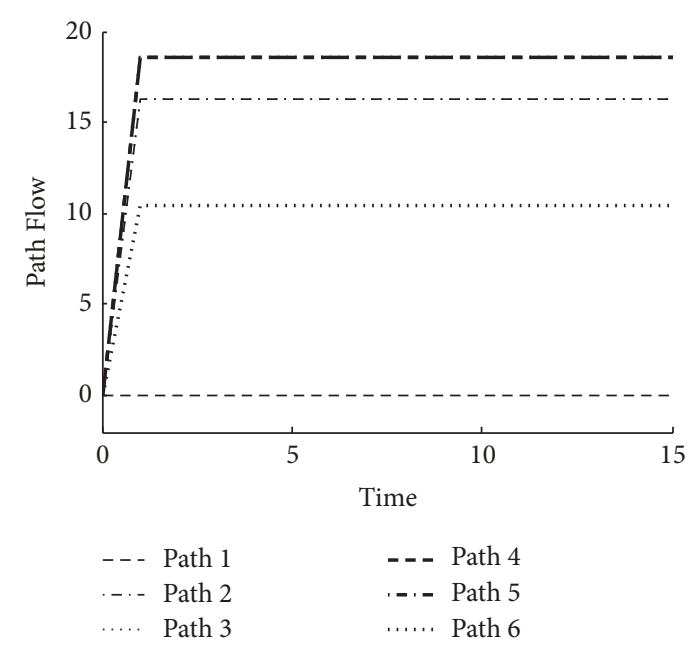

(b) Partial magnification for dynamic regulation process

FIGURE 3: Evolutionary trajectories under single quantity regulation.

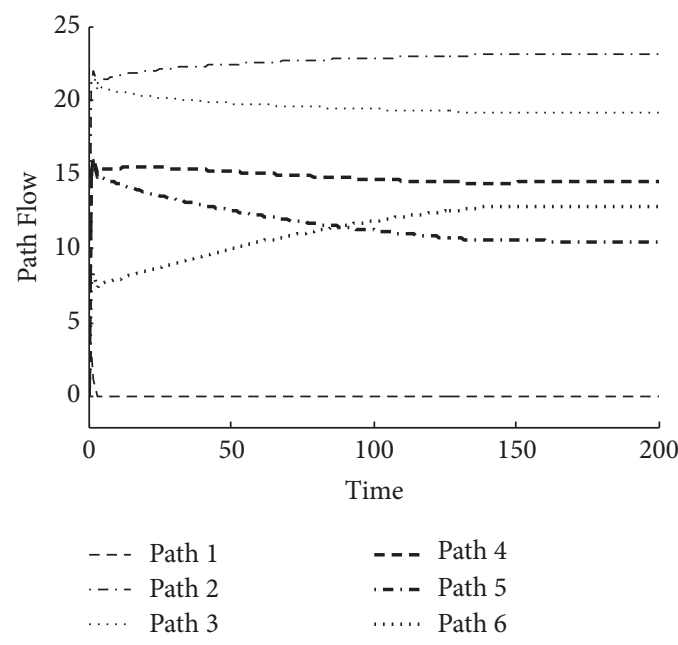

(a) Dynamic regulation process

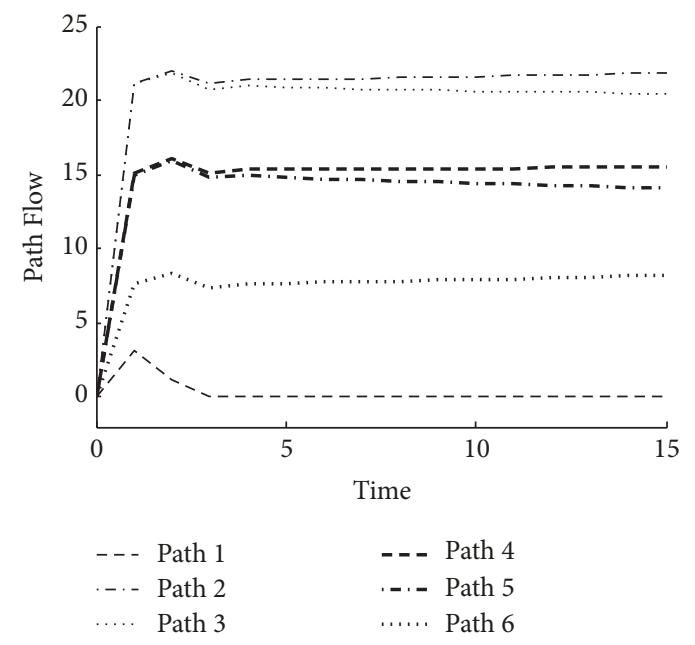

(b) Partial magnification for dynamic regulation process

FIGURE 4: Evolutionary trajectories under price-quantity regulation.

Comparing Tables 3 and 5, it can be found the traffic flow gradually transfers to the paths with larger residual capacity in the price-quantity regulation model which is due to the fact that the impact of regulation capacity on the path choice is considered. Similarly, it can be seen from the results in Tables 4 and 5 that the traffic flow gradually transfers to the paths with less travel time which is due to the fact that the impact of travel time on the path choice is considered. This also shows that the network traffic flow evolution model (25) has the ability to simulate the evolution process of network traffic flow with multiple user class. 


\section{Conclusions}

In this paper, we regarded path-travel time as price and pathresidual capacity as quantity and proposed three types of path choice behavior criteria based on disequilibrium theory in economics. Based on this, we used the method of network tatonnement process to simulate travelers' path choice behavior and established a network traffic flow evolution model. Assuming that link travel time is a positive, continuous, and strictly monotonically increasing function of link flow, pathresidual capacity is a positive, continuous, and strictly monotonically decreasing function of path flow, and travel demand is a continuous, bounded, and strictly monotonic function of minimum travel time and maximum residual capacity, we proved that a unique solution exists in the evolution model and the unique solution is asymptotically stable under certain conditions. The case study demonstrated that the single price regulation, single quantity regulation, and pricequantity regulation can simulate the evolution process of network traffic flow, but there are obvious differences between the evolution process and the final stable state, and pricequantity regulation model can simulate the evolution process of network traffic flow with multiple user class.

Studying the network traffic flow evolution model based on disequilibrium theory can help to understand the evolution process and the final distribution of the network traffic flow under the heterogeneous circumstance of the travelers. This research work will inspire new ideas for designing transportation network schemes and network traffic flow control strategies. An ongoing extension of this study seeks appropriate quantity regulation variables with suitable factors like road technical level, service level. Another future direction is to introduce the traffic flow evolution model in the implementation of traffic network design.

\section{Data Availability}

No data were used to support this study.

\section{Conflicts of Interest}

The authors declare that there are no conflicts of interest regarding the publication of this paper.

\section{Acknowledgments}

This research work is supported by the National Natural Science Foundation of China (Grants nos. 51338002 and 51408058).

\section{References}

[1] J. G. Wardrop, "Some theoretical aspects of road traffic research," in Proceedings of the Institution of Civil Engineers, pp. 72-73, Operational Research Society, 1953.

[2] M. J. Smith, "The stability of a dynamic model of traffic assignment-an application of a method of Lyapunov," Transportation Science, vol. 18, no. 3, pp. 245-252, 1984.

[3] T. L. Friesz, D. Bernstein, N. J. Mehta, R. L. Tobin, and S. Ganjalizadeh, "Day-to-day dynamic network disequilibria and idealized traveler information systems," Operations Research, vol. 42, no. 6, pp. 1120-1136, 1994.

[4] D. Zhang and A. Nagurney, "On the local and global stability of a travel route choice adjustment process," Transportation Research Part B: Methodological, vol. 30, no. 4, pp. 245-262, 1996.

[5] F. Wei, S. Ma, and N. Jia, "A Day-to-Day Route Choice Model Based on Reinforcement Learning," Mathematical Problems in Engineering, vol. 2014, Article ID 646548, 19 pages, 2014.

[6] C.-H. Wen, W.-C. Wang, and C. Fu, "Latent class nested logit model for analyzing high-speed rail access mode choice," Transportation Research Part E: Logistics and Transportation Review, vol. 48, no. 2, pp. 545-554, 2012.

[7] Q. Chen and S. Pan, "Direct formulation and algorithms for the probit-based stochastic user equilibrium traffic assignment problem," Transportation Planning and Technology, vol. 40, no. 7, pp. 757-770, 2017.

[8] L. J. Savage, The Foundations of Statistics, John Wiley \& Sons, Inc., New York, NY, USA, 1954.

[9] P. R. Blavatskyy, "Stochastic expected utility theory," Journal of Risk and Uncertainty, vol. 34, no. 3, pp. 259-286, 2007.

[10] Q. Li, F. Liao, H. J. P. Timmermans, and J. Zhou, "A user equilibrium model for combined activity-travel choice under prospect theoretical mechanisms of decision-making under uncertainty," Transportmetrica A: Transport Science, vol. 12, no. 7, pp. 629-649, 2016.

[11] D. Liu, "Exploring the Impact of Commuter's Residential Location Choice on the Design of a Rail Transit Line Based on Prospect Theory," Mathematical Problems in Engineering, vol. 2014, Article ID 536872, 12 pages, 2014.

[12] G. Wang, S. Ma, and N. Jia, "A combined framework for modeling the evolution of traveler route choice under risk," Transportation Research Part C: Emerging Technologies, vol. 35, pp. 156-179, 2013.

[13] C. G. Chorus, "Regret theory-based route choices and traffic equilibria," Transportmetrica, vol. 8, no. 4, pp. 291-305, 2012.

[14] G. D. M. Ramos, W. Daamen, and S. Hoogendoorn, "Expected utility theory, prospect theory, and regret theory compared for prediction of route choice behavior," Transportation Research Record, vol. 2230, pp. 19-28, 2011.

[15] J. Zhang Y and H. Monden, "Transport demand and supply model based on disequilibrium theory," Journal of the Eastern Asia Society for Transportation Studies, vol. 5, no. 1, pp. 12621277, 2003.

[16] Z. X. Huang, W. B. Fang, and L. X. Wu, "Equilibrium and disequilibrium problems in traffic system," Systems Engineering, vol. 34, no. 9, pp. 113-119, 2016.

[17] E. Avineri, "The effect of reference point on stochastic network equilibrium," Transportation Science, vol. 40, no. 4, pp. 409-420, 2006.

[18] C. G. Chorus, T. A. Arentze, and H. J. P. Timmermans, "A Random Regret-Minimization model of travel choice," Transportation Research Part B: Methodological, vol. 42, no. 1, pp. 1-18, 2008.

[19] Z. Huang and G. He, "Disequilibrium Transportation Planning View," in Proceedings of the Second International Conference on Transportation and Traffic Studies (ICTTS ), pp. 305-310, Beijing, China.

[20] K. Wu, Y. Chen, J. Ma, S. Bai, and X. Tang, "Traffic and emissions impact of congestion charging in the central Beijing urban area: A simulation analysis," Transportation Research Part D: Transport and Environment, vol. 51, pp. 203-215, 2017. 
[21] Y.-C. Chiou and C. Fu, "Responses of drivers and motorcyclists to congestion charge," pp. 2961-2973.

[22] S. Jia, K. Yang, J. Zhao et al., "The traffic congestion charging fee management model based on the system dynamics approach," Mathematical Problems in Engineering, vol. 2017, Article ID 3024898, 13 pages, 2017.

[23] K. Washbrook, W. Haider, and M. Jaccard, "Estimating commuter mode choice: A discrete choice analysis of the impact of road pricing and parking charges," Transportation, vol. 33, no. 6, pp. 621-639, 2006.

[24] J. Simićević, S. Vukanović, and N. Milosavljević, "The effect of parking charges and time limit to car usage and parking behaviour," Transport Policy, vol. 30, pp. 125-131, 2013.

[25] M. Hasan, M. Jha, and A. M. Ben, "Evaluation of ramp control algorithms using microscopic traffic simulation," Transportation Research Part C: Emerging Technologies, vol. 10, no. 3, pp. 229-256, 2002.

[26] J. R. D. Frejo and E. F. Camacho, "Global versus local MPC algorithms in freeway traffic control with ramp metering and variable speed limits," IEEE Transactions on Intelligent Transportation Systems, vol. 13, no. 4, pp. 1556-1565, 2012.

[27] S. J. Berrebi, K. E. Watkins, and J. A. Laval, "A real-time bus dispatching policy to minimize passenger wait on a high frequency route," Transportation Research Part B: Methodological, vol. 81, pp. 377-389, 2015.

[28] J. Wang, G. L. Hu, and X. He, "Bi-level programming model and the solution algorithm for urban bus departure frequency optimization," Systems Engineering, vol. 31, no. 12, pp. 69-73, 2013.

[29] P. Dupuis and A. Nagurney, "Dynamical systems and variational inequalities," Annals of Operations Research, vol. 44, no. 1-4, pp. 9-42, 1993.

[30] H.-J. Cho and M.-C. Hwang, "Day-to-day vehicular flow dynamics in intelligent transportation network," Mathematical and Computer Modelling, vol. 41, no. 4-5, pp. 501-522, 2005.

[31] F. X. Browne, "Sluggish quantity adjustment in a non-clearing market-a disequilibrium econometric application to the loan market," Journal of Applied Econometrics, vol. 2, no. 4, pp. 335349, 1987.

[32] Z. Huang X, J. Cai R, and X. Wu L, "Disequilibrium adjustment mechanism for urban resident travel markets," Journal of Chang'an University, vol. 36, no. 6, pp. 106-110, 2016. 


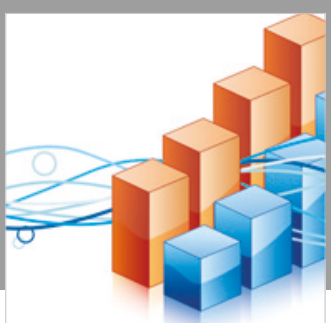

Advances in

Operations Research

\section{-n-m}
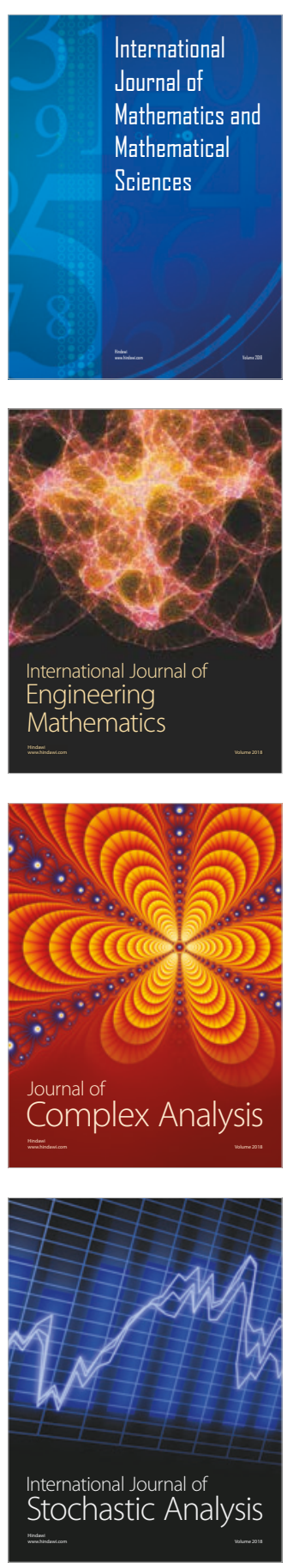
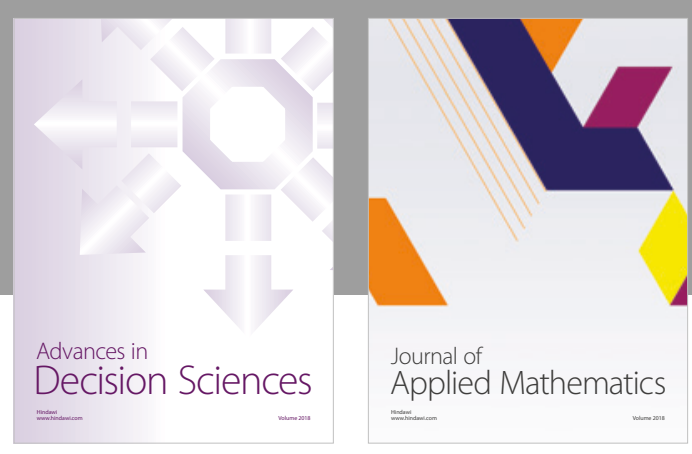

Journal of

Applied Mathematics
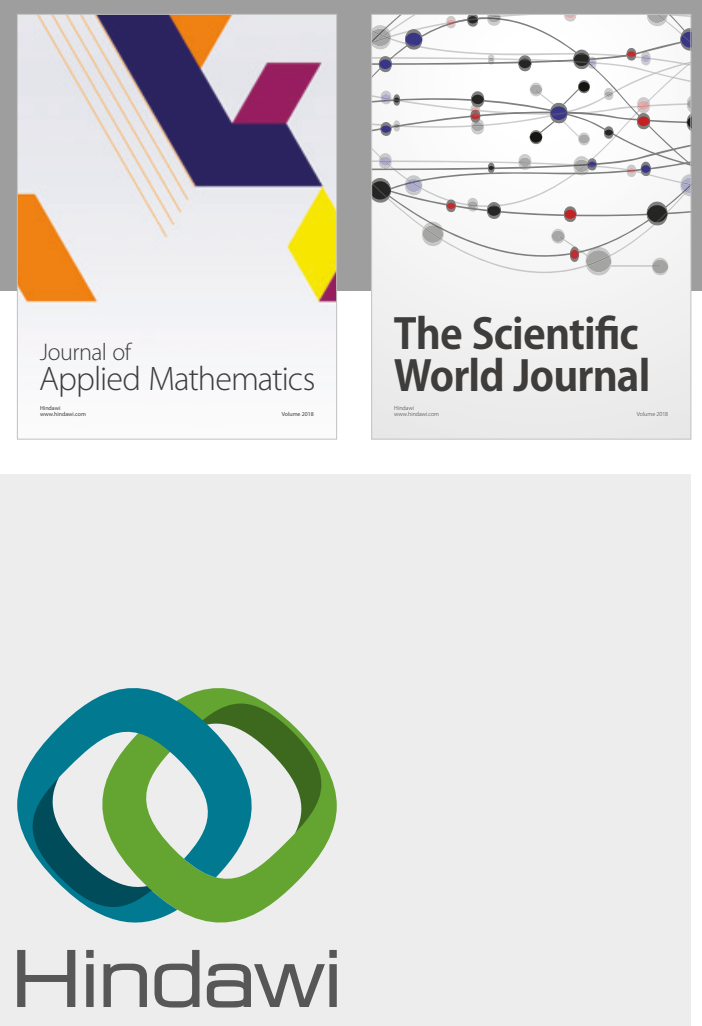

Submit your manuscripts at

www.hindawi.com

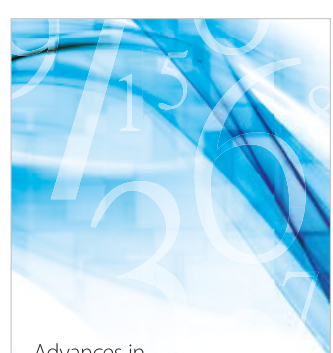

Advances in
Numerical Analysis
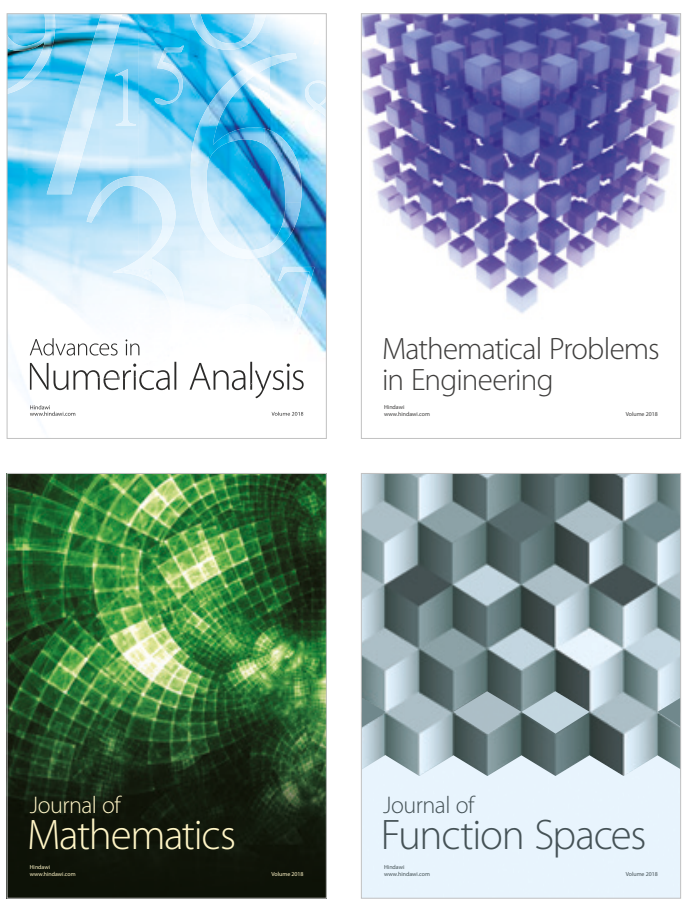

Mathematical Problems in Engineering

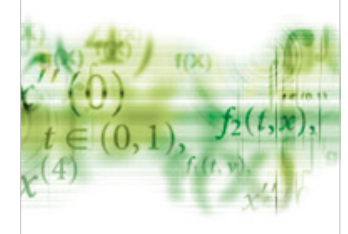

International Journal of

Differential Equations

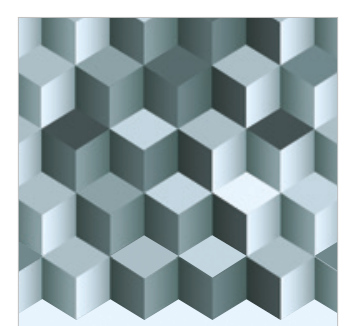

Journal of

Function Spaces

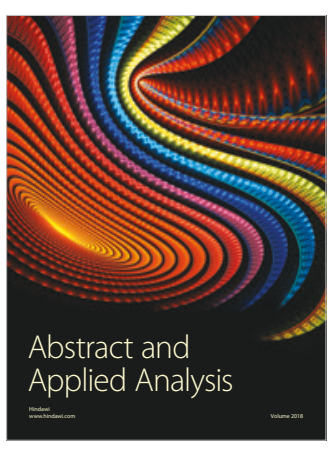

The Scientific

World Journal

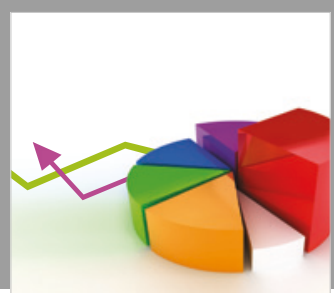

Journal of

Probability and Statistics
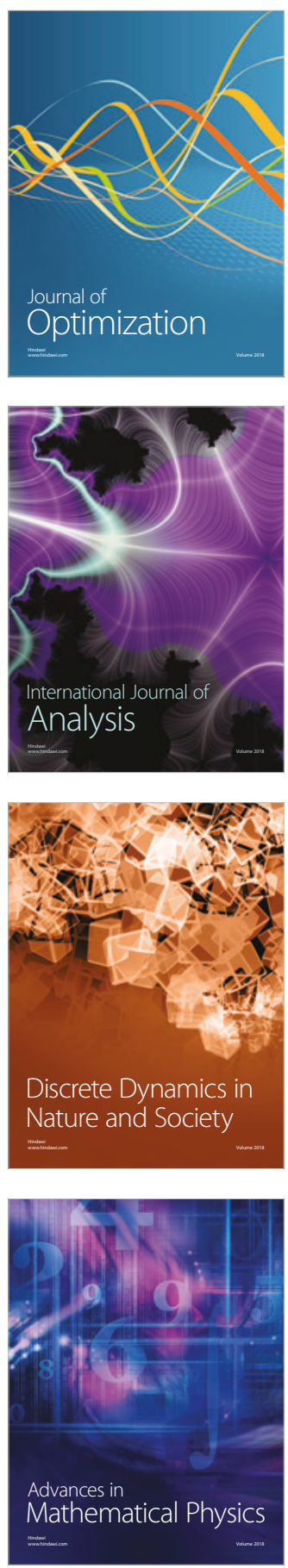